\title{
Disseminated Intraabdominal Echinococcosis: A Case Report
}

\author{
Gangadhar $K^{1}$, Santhosh $D^{2}$, Goel $K^{1}, \operatorname{Rana} S^{1}$, Jain $S^{1}$ \\ ${ }^{1}$ Department of Radiodiagnosis and Imaging, Institute of Medical Sciences, Banaras Hindu \\ University, Varanasi, India, ${ }^{2}$ Department of Pathology, Institute of Medical Sciences, \\ Banaras Hindu University, Varanasi, India
}

\begin{abstract}
Human hydatid disease results from infection with larval form of Echinococcus granulosus. The disseminated intra-peritoneal hydatid disease is a very rare finding. A case of disseminated intra abdominal hydatid disease is presented along with a review of literature and various therapeutic modalities.
\end{abstract}

Keywords: Dissemin, Hydatid cyst, Peritoneal hydatosis, Rectovesical hydatid cyst

\section{Case Report}

A 58-year-old female, belonging to rural north India of poor socio-economic status presented with complaints of cough and respiratory distress since 1 week and the patient had history of anorexia, fatigue, weight loss, abdominal lump and intermittent high-grade fever for 4 months. Her past medical history and family history were unremarkable. Physical examination revealed a diffuse abdominal swelling with lobulations from pelvis to the xiphoid, with a dull note on percussion over it. There was non-tender moderate hepatosplenomegaly.On per rectal examination, a mass was felt from anterior rectal wall but rectal mucosa was free. Review of other systems was normal. On laboratory investigations differential count revealed increase in the eosinophil count $(15 \%)$ and raised ESR. Rest of serum

Correspondence to: Dr. Kiran Gangadhar Department of Radiodiagnosis and Imaging, Institute Of Medical Sciences, Banaras Hindu University, Varanasi, India Email: kirang.585@googlemail.com biochemistry screen revealed normal investigations including normal liver function profile.

Computed tomography of abdomen showed multiple well-defined thin walled cystic lesions with internal septations in a typical "spoke wheel" and "honey comb" pattern noted involving liver, spleen, gastro-splenic ligament, bilateral hepato-renal pouches, pelvis, and rest of the intraperitoneal region. Associated with left pleural effusion suggestive of transdiaphragmatic rupture of cyst into pleural space. These typical findings lead to the diagnosis of disseminated abdominal echinococcosis.

\section{Discussion}

Echinococcosis is a zoonotic disease caused by Echinococcus spp. tapeworms. The definitive hosts, which include dogs, other canids, hyenas and cats, carry the adult tapeworms subclinically. Dogs are particularly important in zoonotic transmission due to their close relationships with humans. Intermediate hosts are initially 

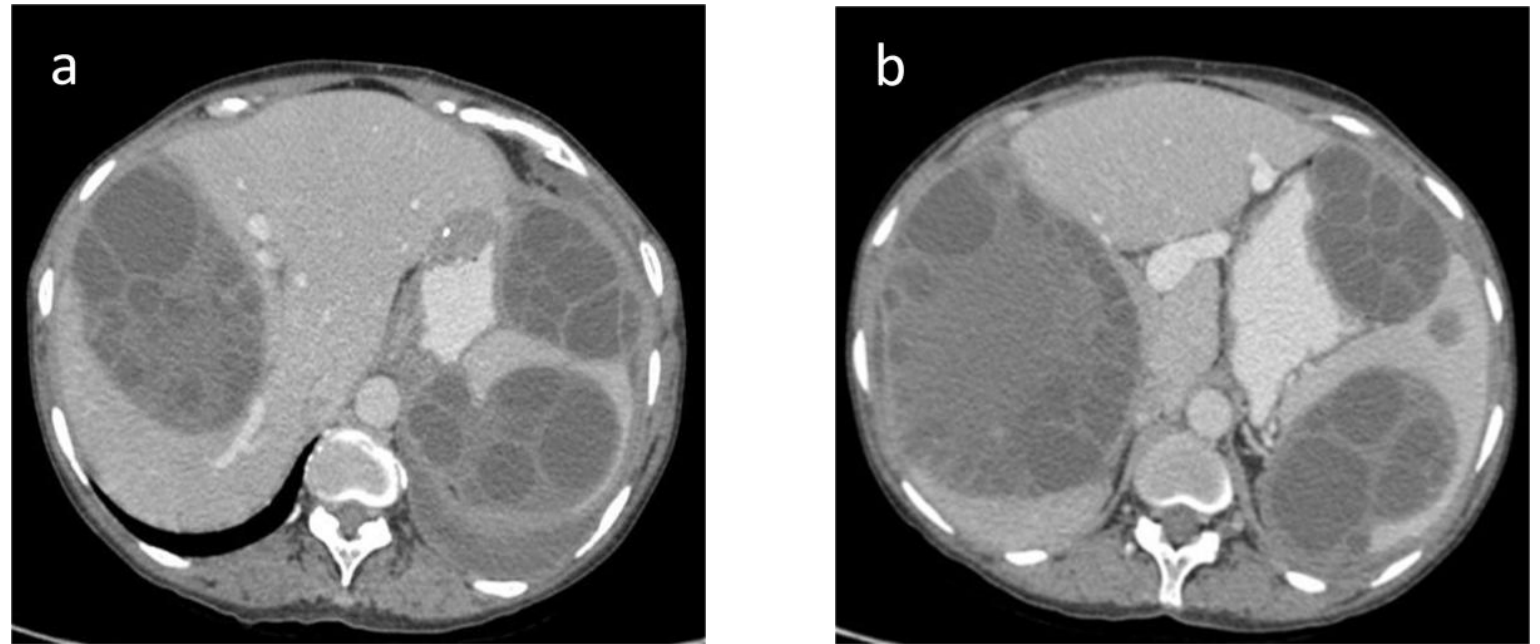

Fig 1: Axial CECT of abdomen showed multiple well-defined thin walled cystic lesions with internal septations and multiple daughter cysts in a typical "spoke wheel" and "honey comb" pattern noted involving liver, spleen, gastro-splenic ligament. Associated with left pleural effusion suggestive of transdiaphragmatic rupture of cyst into pleural space (fig a).
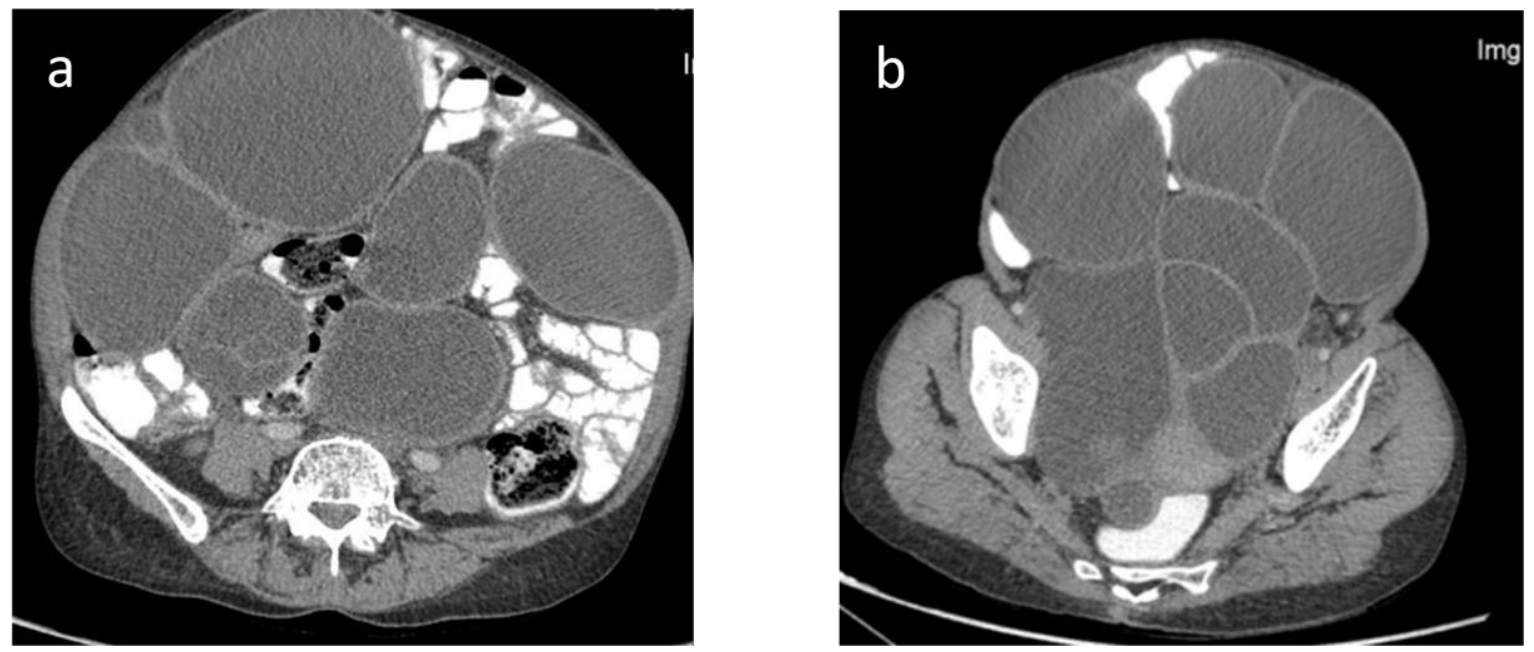

Fig 2: Axial CECT of abdomen showed multiple well-defined thin walled cystic lesions many showing single locule an few showing internal septations in a typical "spoke wheel" and "honey comb" pattern noted involving, bilateral hepato-renal pouches, pelvis, and rest of the intraperitoneal region suggestive of peritoneal dissemination.

asymptomatic; however, the growth of the larvae, which form cysts in vital organs such as the liver and lungs, can lead to illness and death. Echinococcosis is a major public health problem in some countries, and it may be emerging or re-emerging in some areas. Approximately 2-3 million human cases are thought to occur worldwide. ${ }^{1}$

Cystic echinococcosis, the most common form of the disease in people and domesticated animals, is caused by
Echinococcus granulosus sensu lato. Because the larvae of this organism usually develop as discrete single cysts, it is the least severe and most treatable form. Nevertheless, large or multiple cysts may cause irreversible damage to organs, and the rupture or puncture of the cyst can seed multiple organs with larvae or cause anaphylactic reactions. Humans typically become symptomatic many years after infection. Most livestock are slaughtered before the cysts become large enough to cause clinical signs, but if their 
entrails are fed to dogs, it perpetuates the cycle. Animals that live long enough, such as horses, may become ill. In addition, cystic echinococcosis causes economic losses from the condemnation of internal organs at meat inspection. In some cases, it may also result in decreased meat and milk production or decreased value of the fleece due to debilitation. $^{2}$
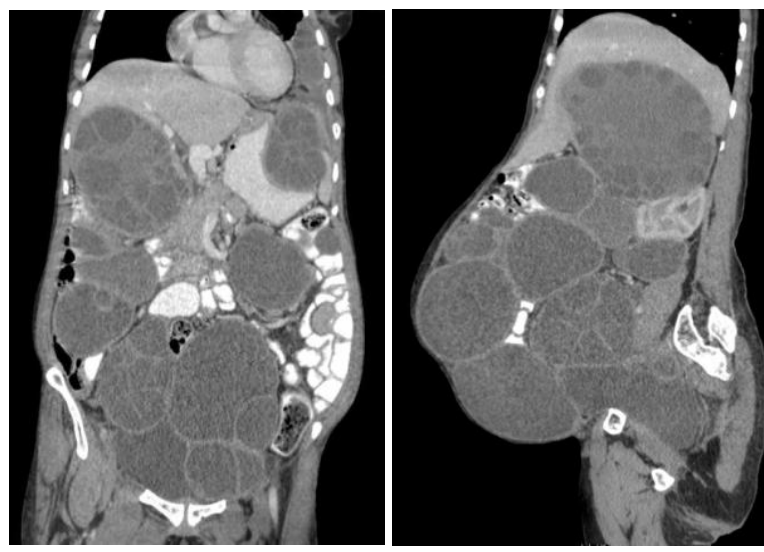

Fig 3: Coronal CECT (fig a) and sagittal CECT (fig b) of abdomen showed multiple well-defined thin walled cystic lesions with internal septations in a typical "spoke wheel" and "honey comb" pattern noted involving liver, spleen, gastro-splenic ligament, bilateral hepato-renal pouches, pelvis, and rest of the intraperitoneal region. Associated with left pleural effusion (fig a) suggestive of transdiaphragmatic rupture of cyst into pleural space.

Peritoneal hydatid disease may grow and occupy the entire peritoneal cavity, simulating a multilobulated mass. This pathological condition is known as encysted peritoneal hydatidosis. Peritoneal hydatid disease represents an uncommon occurrence and its diagnosis is more accurate today due to the new imaging techniques. ${ }^{4} \mathrm{~A}$ double contrast CT scan is $90-100 \%$ accurate for diagnosing secondary echinococcosis and is superior to USG in identifying additional extrahepatic intra abdominal cysts. ${ }^{3} \mathrm{CT}$ is the modality of choice for these patients because it permits imaging of the entire abdomen and pelvis. ${ }^{5}$

The role of medical treatment in hydatid disease is limited. ${ }^{6}$ It is used predominantly as an adjuvant therapy for extensive hepatic or extrahepatic hydatid disease. ${ }^{7}$ Albendazole is the drug of choice. ${ }^{8,9}$ Long term use of this drug is not advised in view of its hepatotoxicity. ${ }^{9}$

Surgery therefore remains the mainstay of treatment for hydatid disease of the liver and is indicated in all patients with symptomatic disease. ${ }^{6,7}$ The goals of surgery for hepatic hydatid disease is to remove all the parasite, to prevent spillage of parasitic daughter cyst and protoscolices and to preserve as much of liver function. Care has to be taken to detect any cyst biliary communication at the time of surgery.

Prevention of echinococcosis focuses primarily on veterinary interventions to control the extent and intensity of infection in definitive host populations, which may indirectly be approached by controlling the prevalence in animal intermediate hosts also.

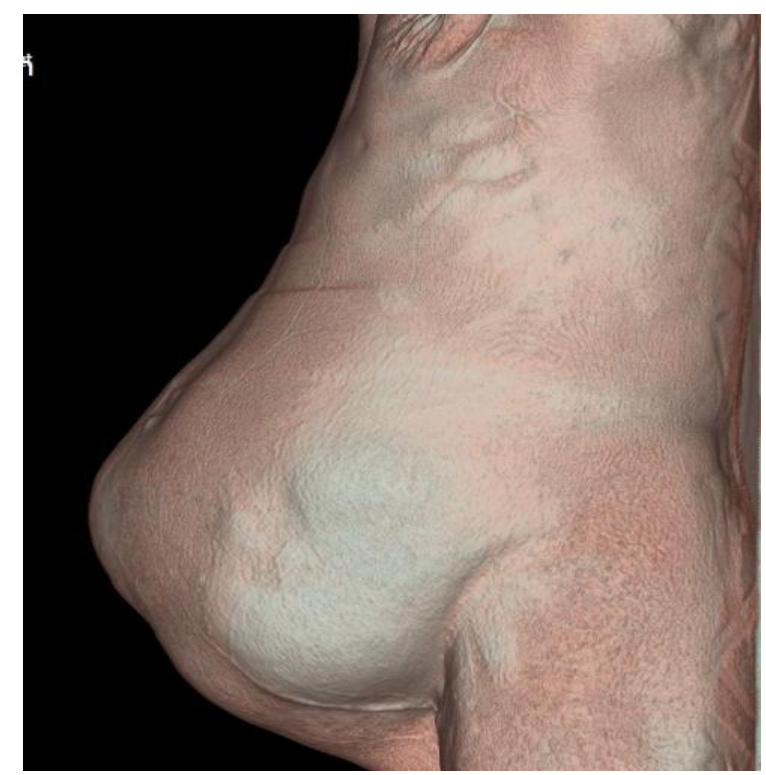

Fig 4a: Volume rendered image of abdomen showing surface lobulations because intraabdominal cysts extending from pelvis to umbilical region with prominent superficial veins. 


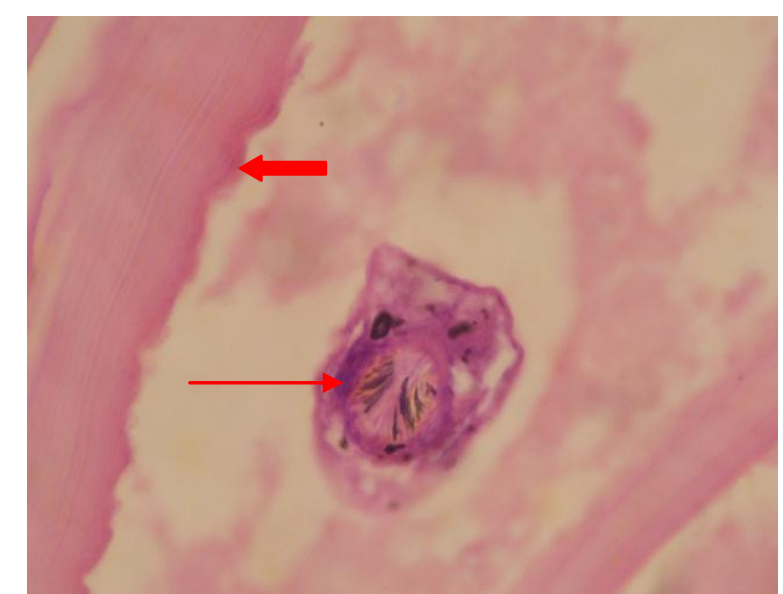

Fig 4b: HPE of the cyst shows lamellated layer (solid arrow) of endocyst wall with protoscolex with hooklets (thin arrow).

The first includes regular pharmacologic treatment and taking sanitary precautions for handling pets or meat to prevent infection and egg excretion. ${ }^{10}$ For the second, a vaccine for ruminant intermediate hosts is in evaluation. Prevention of human infection is strategically very difficult.

\section{Teaching point}

Echinococcosis is one of the commonest parasitic infections of the liver. It can cause a variety of complications in the liver. Rupture into the peritoneal cavity leading to secondary echinococcosis is a difficult problem to manage. Early diagnosis and optimal choice of surgical management reduces the morbidity and mortality associated with this disease.

\section{References}

1. Acha PN, Szyfres B (Pan American Health Organization [PAHO]). Zoonoses and communicable diseases common to man and animals. Volume 3. Parasitoses. $3^{\text {rd }}$ ed. Washington DC: PAHO; 2003. Scientific and Technical Publication No. 580. Hydatidosis; p. 184-199.
2. Beaver PC, Jung RC, Cupp EW. Clinical parasitology. 9th ed. Philadelphia: Lea \& Febiger; 1984. Genus Echinococcus; p. 527-538.

3. Sahu SK, Singh P, Sachan PK,et al. Secondary echinococcosis. Indian J Surg 2006;68:334-5.

4. Tarcoveanu E, Dimofte G, Bradea C,et al. Multiple Peritoneal Hydatid Disease after Rupture of a Multivesicular Hepatic Hydatid Cyst. Case report. J Gastrointestin Liver Dis 2006;15:301-5.

5. Yuksel M, Demirpolat G, Sever A, et al. Hydatid disease involving some Rarelocations in the body: a pictorial essay. Korean J Radiol 2007;8:531-406.

6. Cohen Z, Stone RM, Langer B. Surgical treatment of hydatid disease of the liver. Can J Surg 1976;19:416.

7. Karavias DD, Vagianos CE, Kakkos SK, et al. Preitoneal echinococcosis. World J Surg 1996;20:337.

8. Kern P. Human echinococcosis: Follow up of 23 patients treated with mebendazole. Infection 1983;11:17-24.

9. Morris DL. Preoperative albendazole therapy for hydatid cyst. Br J Surg 1987; 74: 805 .

10. Pawlowski ZS, Eckert J, Vuitton DA, et al. Echinococcosis in humans: clinical aspects, diagnosis and treatment. In: Eckert $\mathrm{J}$ et al., eds. WHO/OIE Manual on echinococcosis inhumans and animals. Paris: WHO/OIE;2001:20-71. 